\title{
The evolution of stars in the Taurus-Auriga T association ${ }^{\star}$
}

\author{
C. Bertout ${ }^{1}$, L. Siess ${ }^{2}$, and S. Cabrit ${ }^{3}$ \\ 1 Institut d'Astrophysique, 98bis Bd. Arago, 75014 Paris, France \\ e-mail: claude.bertout@obspm.fr \\ 2 Institut d'Astronomie et d'Astrophysique, Université Libre de Bruxelles, CP 226, 1050 Brussels, Belgium \\ ${ }^{3}$ LERMA, Observatoire de Paris, 61 Av. de l'Observatoire, 75014 Paris, France
}

Received 13 July 2007 / Accepted 20 August 2007

\section{ABSTRACT}

\begin{abstract}
In a recent study, individual parallaxes were determined for many stars of the Taurus-Auriga $\mathrm{T}$ association that are members of the same moving group. We use these new parallaxes to re-address the issue of the relationship between classical T Tauri stars (CTTSs) and weak-emission line T Tauri stars (WTTSs). With the available spectroscopic and photometric information for 72 individual stars or stellar systems among the Taurus-Auriga objects with known parallaxes, we derived reliable photospheric luminosities, mainly from the $I_{\mathrm{c}}$ magnitude of these objects. We then studied the mass and age distributions of the stellar sample, using pre-main sequence evolutionary models to determine the basic properties of the stellar sample. Statistical tests and Monte Carlo simulations were then applied to studying the properties of the two T Tauri subclasses. We find that the probability of CTTS and WTTS samples being drawn from the same parental age and mass distributions is low; CTTSs are, on average, younger than WTTSs. They are also less massive, but this is due to selection effects. The observed mass and age distributions of both T Tauri subclasses can be understood in the framework of a simple disk evolution model, assuming that the CTTSs evolve into WTTSs when their disks are fully accreted by the stars. According to this empirical model, the average disk lifetime in Taurus-Auriga is $4 \times 10^{6}\left(M_{*} / M_{\odot}\right)^{0.75} \mathrm{yr}$.
\end{abstract}

Key words. stars: formation - stars: pre-main sequence - stars: circumstellar matter

\section{Introduction}

In a recent work focusing on the kinematic properties of the Taurus-Auriga T association, (Bertout \& Genova 2006, Paper I hereafter) analyzed the proper motions catalogued in Ducourant et al. (2005). They identified a (minimum) moving group of 94 stars or stellar systems sharing the same spatial velocity and derived kinematic parallaxes for 67 of those objects. These co-moving stars define the T-association as regards its kinematics.

In this Letter, we use these new parallaxes to re-address the question of the relationship between the two subgroups of T Tauri stars (TTSs): (a) the CTTSs, which were detected primarily in the course of $\mathrm{H} \alpha$ surveys of dark clouds and are actively accreting from their circumstellar disks, and (b) the WTTSs that were identified both from $\mathrm{H} \alpha$ and CaII line emission surveys and X-ray surveys. They are magnetically active, young stars that show no spectroscopic evidence for accretion and are not usually associated with circumstellar disks.

The exact relationship between CTTSs and WTTSs remains elusive. Both species share the same region of the HertzsprungRussell diagram (HRD), and the most obvious difference between them is their somewhat different location with respect to the molecular clouds with which they are associated. The new parallaxes derived in Paper I have confirmed the previous finding (e.g., Feigelson 1996, and references therein) that the distribution in space of optically detected TTSs and X-ray selected WTTSs is different. CTTSs are usually found in the inner regions of the molecular clouds, while WTTSs tend to be found on

$\star$ Table 4 is only available in electronic form at http: //www . aanda.org the outskirts of starforming regions. However, optical WTTSs were originally searched for only in the immediate vicinity of the molecular clouds and were therefore found in roughly the same regions as CTTSs. In contrast, X-ray selected WTTSs were mainly discovered in the course of large-scale X-ray surveys, so some of them were found far away from the central parts of starforming regions. More recent searches for additional optical WTTSs did not lead to the discovery of many previously unnoticed WTTSs in the Taurus-Auriga area. While successful at discovering new CTTSs in Taurus, the Spitzer observations reported by Luhman et al. (2006) show that the previous census (Gomez et al. 1993) of Taurus objects down to $V \approx 14-$ which is also the approximate limiting magnitude of the Ducourant et al. (2005) sample - was $80 \%$ complete.

To explain the existence of both CTTSs and WTTSs, one usually postulates that newborn stars display a wide range of disk masses and that their accretion or dispersal requires a correspondingly wide range of timescales, which justifies the stars intermingling in the HRD (e.g., Simon \& Prato 1995). In other terms, it is only the evolutionary status of their circumstellar disks that distinguishes the subgroups. However, this explanation does not rest on firm observational support yet. We thus re-address here the issue of the respective evolutionary status of CTTSs and WTTSs. We show that there are in fact significant differences in their ages and that their mass and age distributions can be understood within a simple evolutionary framework.

\section{Photospheric luminosities of moving-group stars}

In Paper I, we derived parallaxes for 30 CTTSs or CTTS systems, 36 WTTSs or WTTS systems, and 1 Herbig Be star. The 


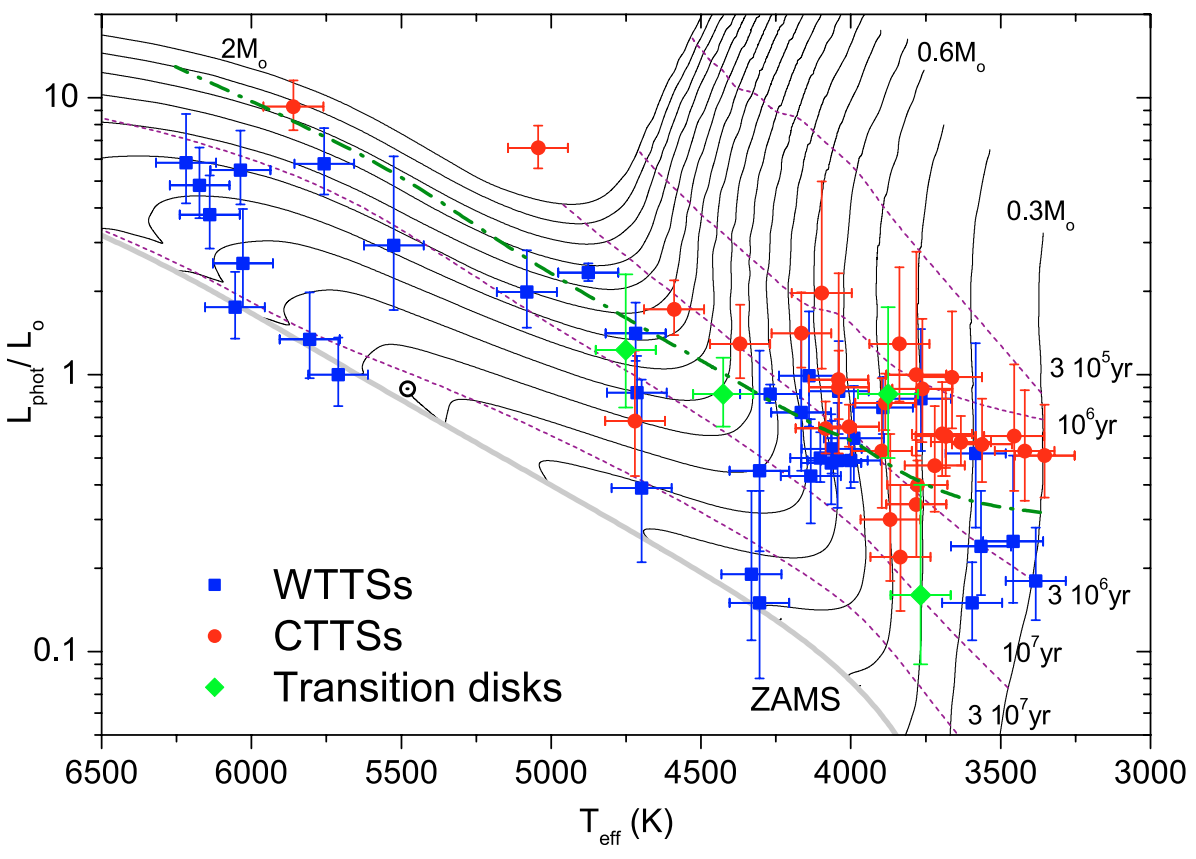

Fig. 1. HRD of the moving group stars. Red dots denote CTTSs, blue squares mark WTTSs, and green diamonds indicate the stars with transition disks (DI Tau, DM Tau, GM Aur, and Lk Ca 15). The error bars indicate the $1 \sigma$ uncertainties on $L_{\text {phot }}$ and $T_{\text {eff }}$. The solid black lines are evolutionary tracks, computed with $Y=0.277$ and $Z=0.02$, for stars with masses ranging from 0.3 to $2 M_{\odot}$ with a mass increment of $0.1 M_{\odot}$. The current position of the Sun as computed with these parameters is shown as a solar symbol. Note that the parameters chosen for the computation of pre-main sequence evolution tracks are not solar, so that the properties of the computed $1 M_{\odot}$ model do not exactly correspond to those of the actual Sun. The dashed lines are isochrones for the ages indicated in the figure, while the heavy dark-green dash-dotted line shows the disk lifetime $\tau_{\mathrm{d}}$ as a function of stellar mass as defined by Eq. (1). respective numbers of CTTSs and WTTSs given here correct those given in Sect. 6.3 of Paper I, where some objects were misidentified. The conclusions of Paper I are not affected by this error. We mainly used the photometric and spectroscopic data given by Kenyon \& Hartmann (1995) in their thorough investigation of the Taurus-Auriga population. The visual extinction values were also taken mainly from that work. We supplemented this material with newer data for WTTSs that were discovered after 1995 and for a number of system components that were resolved by recent high angular-resolution observations. To avoid a selection bias, we did not include the components of stellar systems with spectral types later than M4 or derived luminosities lower than $\approx 0.15 L_{\odot}$; these values correspond to the coolest and faintest, apparently single objects found in the original sample. For reasons discussed by Cieza et al. (2005), we also excluded those CTTS components for which only $J$ or $K$ flux measurements are available. Our final sample, including the resolved components of multiple systems that fulfill the above criteria, comprises 33 CTTSs and 38 WTTSs.

The stellar luminosities of all CTTSs were computed from their $I_{C}$ flux, because the contribution of excess emission is smallest in that filter (see Cieza et al. 2005) and from the appropriate bolometric correction, as tabulated for various spectral types in Kenyon \& Hartmann (1995). For the WTTSs, we derived the luminosities either from the $I_{\mathrm{C}}$ flux when available or from the $V$ flux. Since WTTSs have negligible excesses in the optical range, both procedures should be equivalent. Stars for which both measurements were available indeed give the same results to within a few percent. In a few cases of WTTSs lacking visual-extinction determination, we computed an approximate value by requiring that the $V$ and the $I_{C}$ (when available) or 2MASS $J$ flux measurements give the same stellar luminosity. The uncertainties on the new parallaxes dominate the luminosity error budget so strongly that other sources of error can be neglected in a first approximation.

Finally, we built two control samples for the same objects by (a) computing the luminosities as explained above but assuming that the stars were all located at the post-Hipparcos average distance of $139_{-10}^{+12} \mathrm{pc}$ derived for Taurus-Auriga by
Bertout et al. (1999) (called sample CS1 hereafter) and (b) computing the luminosities from the 2MASS $J$ flux and assuming the same distance of $139_{-10}^{+12} \mathrm{pc}$ for all stars (sample CS2 hereafter). The CS1 sample allows us to gauge the effect of the new parallaxes, while CS2 allows for a direct comparison of our result with those of Kenyon \& Hartmann (1995).

\section{HRD of the moving group}

We used the grid of pre-main sequence evolutionary tracks computed by Siess et al. (2000) to derive the masses and age of our stars along with error bars due to the uncertainty on the luminosities and effective temperatures (we assumed a $\pm 100 \mathrm{~K}$ uncertainty for all spectral types). Online Table 4 gives the basic stellar properties of our sample and their uncertainties.

It is apparent in the HRD of the moving group plotted in Fig. 1 that the CTTSs appear on average younger and less massive than WTTSs. This is confirmed by the KolmogorovSmirnov (K-S) statistics for the masses and ages of the data sample shown in Table 1. As discussed by Siess (2001), pre-main sequence evolutionary tracks computed by different groups and using different input physics yield different masses and ages for a given pre-main sequence position in the HRD. The uncertainties on $T_{\text {eff }}$ and $L_{\text {phot }}$ adopted in this study are large enough to offset the age and mass uncertainties that arise from the physical approximations made in our evolutionary code, but do not account for possible systematic effects, such as those introduced by the different shapes of evolutionary tracks obtained by different investigators. To assess the robustness of the mass and age determinations from our models, we thus re-computed the masses and ages of both TTS subgroups by using the D'Antona \& Mazzitelli (1997) tracks and isochrones, and found that the resulting K-S probabilities for the CTTSs and WTTSs to be drawn from the same mass and age parental distributions are respectively $8.3 \times 10^{-3}$ and $6.6 \times 10^{-5}$. This result increases our confidence that the differences between CTTSs and WTTSs reported here are not an artefact caused by our evolutionary tracks. We nevertheless caution that the mass and age values given in 
Table 1. Probabilities that the distributions of stellar properties of CTTSs and WTTSs are drawn from the same parent distribution.

\begin{tabular}{lc}
\hline \hline \multicolumn{2}{l}{ Derived masses for data and control samples } \\
\hline Mass & $(3.9 \pm 3.4) \times 10^{-4}$ \\
Mass $(\mathrm{CS} 1)$ & $(7.8 \pm 5.1) \times 10^{-4}$ \\
Mass $(\mathrm{CS} 2)$ & $(2.4 \pm 2.1) \times 10^{-4}$ \\
\hline \multicolumn{2}{c}{ Derived ages for data and control samples } \\
\hline Age & $(1.4 \pm 1.2) \times 10^{-4}$ \\
Age $(\mathrm{CS} 1)$ & $(3.7 \pm 2.0) \times 10^{-3}$ \\
Age $(\mathrm{CS} 2)$ & $(1.4 \pm 0.8) \times 10^{-2}$ \\
\hline
\end{tabular}

Table 2. Average and median masses and ages for TTS samples.

\begin{tabular}{lcccc}
\hline \hline & $\bar{M} \pm \sigma_{\bar{M}}$ & Med. $M$ & $\overline{\overline{\log t}} \pm \sigma_{\overline{\log t}}$ & Med. $\log t$ \\
& $M_{\odot}$ & $M_{\odot}$ & $t$ in yr & $t$ in yr \\
\hline CTTSs & $0.72 \pm 0.44$ & 0.57 & $6.37 \pm 0.37$ & 6.28 \\
WTTSs & $0.97 \pm 0.40$ & 0.80 & $6.82 \pm 0.44$ & 6.74 \\
\hline
\end{tabular}

Table 2 and the average disk lifetime derived in Sect. 4 depend on the evolutionary models used in this investigation.

The difference in average masses between the two TTS subgroups is due to an observational selection effect, since the luminosity limit of $\approx 0.15 L_{\odot}$ for our sample precludes any star with a mass lower than $\approx 0.4 M_{\odot}$ being older than $\approx 5 \times 10^{6} \mathrm{yr}$. Because this is an age range for which we expect to have more WTTSs than CTTSs, the mass distribution of WTTSs is skewed toward high masses. This is confirmed by a K-S test showing that the probability is 0.27 that CTTSs and WTTSs more massive than $0.7 M_{\odot}$ are drawn from the same distribution.

The age distributions for CTTSs and WTTSs do not suffer from such a bias, since the missing low-mass WTTSs would only reinforce the age difference between CTTSs and WTTSs suggested by the K-S statistics of Table 1 . Note that the probability that the age distributions are drawn from the same parental distribution is $\sim 10^{-3}$ for the CS1 control sample and $\approx 10^{-2}$ for the CS2 sample. This second value is less significant by two orders of magnitude than the K-S probability found for our data sample using the new parallaxes $\left(\approx 10^{-4}\right)$, but it agrees with what Kenyon \& Hartmann (1995) found in their own study.

We performed Monte-Carlo (MC) simulations to assess the significance of this finding. We first constructed 1000 realizations of our dataset by varying the effective temperatures and computed luminosities within their uncertainties, which we assumed to be normally distributed. We then computed the CTTS and WTTS age distributions for each realization and computed their K-S statistics. The result is a histogram of K-S probabilities for the various realizations of the data set. We repeated this procedure for our two control samples. As a final test, we constructed one more control sample by randomly drawing the effective temperature and stellar luminosity of individual stars within their observed (non-normal) distributions and computing masses and ages of our sample using these values. The histogram of K-S probabilities for this "random" dataset thus allows us to check the validity of the K-S test when used with our non-normal dataset. Figure 2 displays the 4 resulting histograms of K-S probabilities on the same logarithmic scale. The data and random sample histograms have very little overlap, which confirms the significance of the age difference between CTTSs and WTTSs. Age differences are also apparent in the CS1 and CS2 samples,

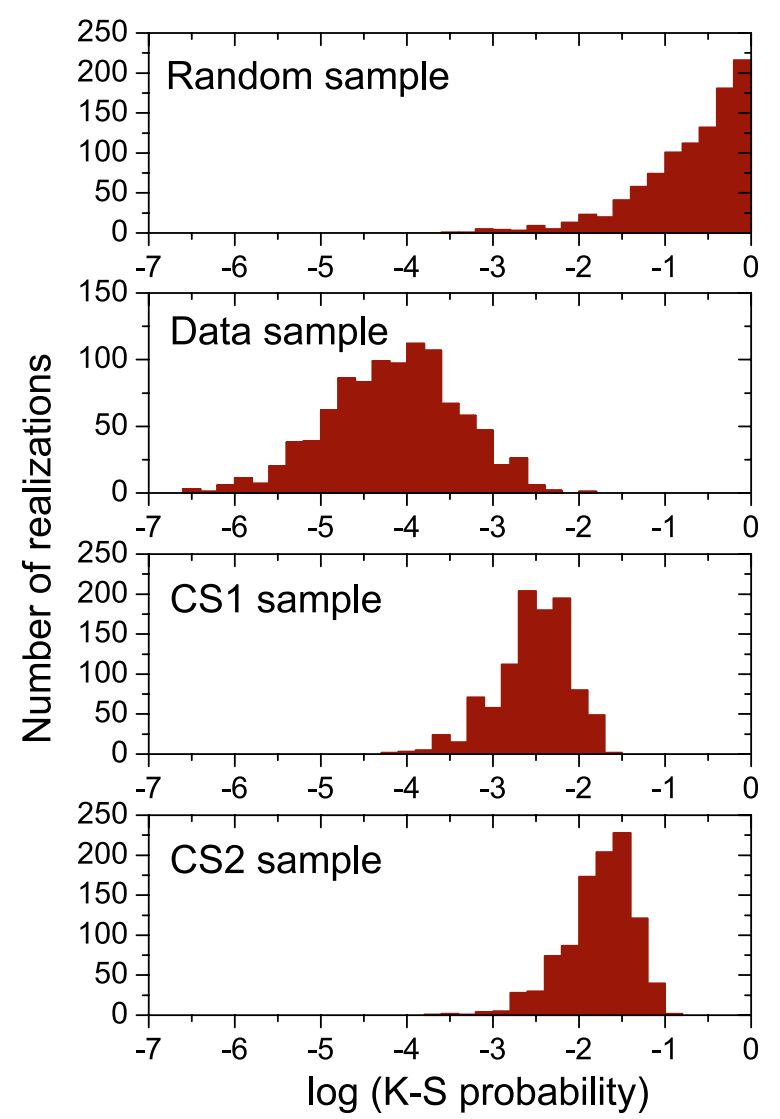

Fig. 2. K-S probability histograms from Monte Carlo simulations for the age distributions of CTTSs and WTTSs (see text for details).

but with lower significance. Clearly, the individual parallaxes of moving-group stars used in this investigation allow for a better differentiation of the two subgroups, even though the large parallax uncertainties act to broaden the probability histogram when compared to the control samples.

\section{The evolution from CTTSs to WTTSs}

With this first indication that a distinction between CTTSs and WTTSs can be seen in the HRD, we were encouraged to look for a simple empirical model that would explain the evolution from CTTS to WTTS based on disk evolution. We thus assumed that the mass of a T Tauri disk in units of $M_{\odot}$ is given by $M_{\mathrm{d}}=\alpha\left(M_{*} / M_{\odot}\right)^{\beta}$ and that the accretion rate is given by $\dot{M}_{\mathrm{d}}=\gamma\left(M_{*} / M_{\odot}\right)^{2.1} M_{\odot} / \mathrm{yr}$, in agreement with current observations over a wide mass range (Muzerolle et al. 2003; Natta et al. 2005). From there, we derived the disk lifetime $\tau_{\mathrm{d}}$ (in yr)

$\log \tau_{\mathrm{d}}=\log (\alpha / \gamma)+(\beta-2.1) \log \left(M_{*} / M_{\odot}\right)$.

For a given combination of $\alpha / \gamma$ and $\beta$, we then compared the age $t_{*}$ of each star - as derived from its evolutionary track - to the disk age $\tau_{\mathrm{d}}\left(M_{*}\right)$. We then assumed that the star was a CTTS whenever $t_{*} \leq \tau_{\mathrm{d}}\left(M_{*}\right)$ or a WTTS whenever $t_{*}>\tau_{\mathrm{d}}\left(M_{*}\right)$. Once we assigned a type to all stars in the sample, we compared the resulting distributions of model CTTS and WTTS masses to the observed mass distributions. Using the K-S statistics to indicate goodness-of-fit, we looked for the best possible match between the mass distributions while varying the parameters in the range $5 \leq \log (\alpha / \gamma) \leq 8$ and $-4 \leq \beta \leq 4$. Much to our surprise - since our model is so simple - we found excellent statistical agreement 
Table 3. Number of MC realizations with K-S probabilities higher than 0.9995 (see text) and average values of the parameters (with their standard deviations) for 1000 realizations of each investigated sample.

\begin{tabular}{lccccc}
\hline \hline & $N\left(p_{\mathrm{K}-\mathrm{S}}>0.9995\right)$ & $\overline{\log (\alpha / \gamma)_{\mathrm{CTTSs}}} \pm \sigma$ & $\overline{\beta_{\mathrm{CTTSs}}} \pm \sigma$ & $\overline{\log (\alpha / \gamma)_{\mathrm{WTTS}}} \pm \sigma$ & $\overline{\beta_{\mathrm{WTTS}} \pm \sigma}$ \\
\hline Data & $97.6 \%$ & $6.63 \pm 0.05$ & $2.85 \pm 0.21$ & $6.58 \pm 0.08$ & $2.87 \pm 0.21$ \\
CS1 & $59.2 \%$ & $6.62 \pm 0.03$ & $2.90 \pm 0.15$ & $6.58 \pm 0.05$ & $2.76 \pm 0.22$ \\
CS2 & $5.2 \%$ & $6.33 \pm 0.06$ & $2.21 \pm 0.17$ & $6.26 \pm 0.04$ & $2.03 \pm 0.18$ \\
Random & $8.0 \%$ & $6.78 \pm 0.13$ & $3.89 \pm 0.61$ & $6.76 \pm 0.12$ & $3.89 \pm 0.54$ \\
\hline
\end{tabular}

between the observed and modeled mass distributions for both CTTSs and WTTSs in a single and very well-defined range of parameters around $\log (\alpha / \gamma) \approx 6.6$ and $\beta \approx 2.9$. The resulting $\tau_{\mathrm{d}}$ locus in the HRD is shown in Fig. 1.

To examine this in more detail, we again resorted to MC simulations, scanning the $(\alpha / \gamma, \beta)$ parameter space for each of the 1000 realizations of our data sample. The results are summarized in Table 3 with the results found for the CS1, CS2, and random samples (see above). Again, the derived $\alpha / \gamma$ and $\beta$ are confined to a single and well-defined range of values. We find that $8 \%$ of the random sample have a K-S probability 0.9995 or higher that the modeled and observed CTTS and WTTS mass distributions are drawn from the same respective parental distributions. In contrast, this probability is higher than 0.9995 in $97.6 \%$ of all realizations of our actual data set, and the computations yield very similar parameter values for both CTTSs and WTTSs. Simulations using the CS1 sample gave a lower percentage of high-probability results, but the derived parameter values are very close to those found with the original dataset. Our results thus remain valid if we use the Taurus average parallax value instead of the individual parallaxes. The situation is much less favorable when using the CS2 sample, since the number of highprobability realizations drops down to a value similar to the one for the random sample. This confirms that using the flux in $J$ as a proxy for the photospheric luminosity of CTTSs blurs the differences between CTTS and WTTS properties.

We conclude that the observed distributions of ages and masses in the Taurus-Auriga moving group can be explained by assuming that a CTTS evolves into a WTTS when the disk is fully accreted by the star. Such an evolution has been hypothesized for a long time, but it is the first time that observational evidence unambiguously supports this scenario. The average disk lifetime in Taurus-Auriga is found to be $4 \times 10^{6}\left(M_{*} / M_{\odot}\right)^{0.75} \mathrm{yr}$ in the framework of our heuristic. If we assume an average mass accretion rate of $10^{-8} M_{\odot} / \mathrm{yr}$ for a typical $1 M_{\odot}$ CTTS, the disk mass is given in the same framework by $M_{\mathrm{d}}=0.04\left(M_{*} / M_{\odot}\right)^{2.85}$.

The results derived above are only valid in a statistical sense. As seen in Fig. 1, some CTTSs are older than the lifetime of their disk, while some WTTSs are younger; these stars are thus misidentified by our model. The misidentified CTTSs are the oldest members of the subgroup: CW Tau, GO Tau, DM Tau, VY Tau, IP Tau, LkCa 15, and GM Aur. Only 2 CTTSs (GO Tau and CW Tau) out of 33 are older by more than $1 \sigma$ than their expected disk lifetime $\tau_{\mathrm{d}}$. Mass accretion for DM Tau, GM Aur, and IP Tau range from $8 \times 10^{-10}$ to $9 \times 10^{-9} M_{\odot} / y r$ (Valenti et al. 1993; Gullbring et al. 1998), thus indicating rather mild accretion activity. Furthermore, DM Tau, GM Aur, and LkCa 15 are believed to be transition objects that are currently clearing their disks (Calvet et al. 2005; Piétu et al. 2006). Properties of these objects are thus compatible with those of CTTSs approaching the end of their disk-accretion phase. The exception that our model does not account for is CW Tau, which harbors a jet and has a mass accretion rate of $6 \times 10^{-8} M_{\odot} / \mathrm{yr}$
(Mohanty et al. 2005). The misidentified WTTSs are also the youngest of the sample: LkCa 14, V928 Tau, DI Tau, IW Tau, V827 Tau, and RX J0432.8+1735. Again, most of these objects have ages within $1 \sigma$ of their respective $\tau_{\mathrm{d}}$. While most of them are rather unremarkable WTTSs, Meyer et al. (1997) have proposed that DI Tau is a transitional object surrounded by a fastevolving disk.

As a final remark, we note that the relationship between disk mass and stellar mass derived above leads to very low masses for disks surrounding brown dwarfs and will thus be falsified by the first observation of a massive brown dwarf disk unless the masses of CTTS disks have been largely underestimated, which remains a possibility if substantial grain growth takes place in these disks (cf. Natta et al. 2007). As mentioned by Alexander \& Armitage (2006) and Hartmann et al. (2006), a steep relationship between disk and stellar masses would also help us understand the dependence of the mass accretion rate on the stellar mass.

Acknowledgements. This research made use of the Centre de Données de Strasbourg facilities and of the NASA/ IPAC Infrared Science Archive.

\section{References}

Alexander, R. D., \& Armitage, P. J. 2006, ApJ, 639, L83

Bertout, C., \& Genova, F. 2006, A\&A, 460, 499

Bertout, C., Robichon, N., \& Arenou, F. 1999, A\&A, 352, 574

Calvet, N., D'Alessio, P., Watson, D. M., et al. 2005, ApJ, 630, L185

Cieza, L. A., Kessler-Silacci, J. E., Jaffe, D. T., Harvey, P. M., \& Evans, II, N. J. 2005, ApJ, 635, 422

D’Antona, F., \& Mazzitelli, I. 1997, Mem. Soc. Astron. It., 68, 807 Ducourant, C., Teixeira, R., Périé, J. P., et al. 2005, A\&A, 438, 769 Feigelson, E. D. 1996, ApJ, 468, 306

Ghez, A. M., White, R. J., \& Simon, M. 1997, ApJ, 490, 353

Gomez, M., Hartmann, L., Kenyon, S. J., \& Hewett, R. 1993, AJ, 105, 1927 Gullbring, E., Hartmann, L., Briceno, C., \& Calvet, N. 1998, ApJ, 492, 323

Hartmann, L., D’Alessio, P., Calvet, N., \& Muzerolle, J. 2006, ApJ, 648, 484 Kenyon, S. J., \& Hartmann, L. 1995, ApJS, 101, 117

Leinert, C., Zinnecker, H., Weitzel, N., et al. 1993, A\&A, 278, 129

Luhman, K. L., Whitney, B. A., Meade, M. R., et al. 2006, ApJ, 647, 1180

Meyer, M. R., Beckwith, S. V. W., Herbst, T. M., \& Robberto, M. 1997, ApJ, 489, L173

Mohanty, S., Jayawardhana, R., \& Basri, G. 2005, ApJ, 626, 498

Muzerolle, J., Hillenbrand, L., Calvet, N., Briceño, C., \& Hartmann, L. 2003, ApJ, 592, 266

Natta, A., Testi, L., Randich, S., \& Muzerolle, J. 2005, Mem. Soc. Astron. It., 76,343

Natta, A., Testi, L., Calvet, N., et al. 2007, in Protostars and Planets V, ed. B. Reipurth, D. Jewitt, \& K. Keil, 767

Piétu, V., Dutrey, A., Guilloteau, S., Chapillon, E., \& Pety, J. 2006, A\&A, 460, L43

Siess, L. 2001, in From Darkness to Light: Origin and Evolution of Young Stellar Clusters, ed. T. Montmerle, \& P. André, ASP Conf. Ser., 243, 581

Siess, L., Dufour, E., \& Forestini, M. 2000, A\&A, 358, 593

Simon, M., \& Prato, L. 1995, ApJ, 450, 824

Valenti, J. A., Basri, G., \& Johns, C. M. 1993, AJ, 106, 2024

Walter, F. M., Brown, A., Mathieu, R. D., Myers, P. C., \& Vrba, F. J. 1988, AJ, 96, 297

Welty, A. D. 1995, AJ, 110, 776

White, R. J., Ghez, A. M., Reid, I. N., \& Schultz, G. 1999, ApJ, 520, 811

Wichmann, R., Torres, G., Melo, C. H. F., et al. 2000, A\&A, 359, 181

Woitas, J., Leinert, C., \& Köhler, R. 2001, A\&A, 376, 982 
C. Bertout et al.: The evolution of stars in the Taurus-Auriga T association, Online Material $p 1$

\section{Online Material}


Table 4. Observational and derived properties of moving-group stars used in this study.

\begin{tabular}{|c|c|c|c|c|c|c|c|c|c|}
\hline Star & Type $^{1}$ & Sp. type & $T_{\text {eff }}$ & $A_{\mathrm{V}}$ & $L_{\text {star }} / L_{\odot}$ & Filter $^{2}$ & $M_{\text {star }} / M_{\odot}$ & $\log t \pm \sigma_{\log t}(t$ in yr $)$ & Ref. $^{3}$ \\
\hline NTTS $035120+3154 \mathrm{SW}$ & $\mathrm{x}$ & G0 & 6030 & 0.87 & $2.52_{-0.77}^{+1.44}$ & $I$ & $1.27 \pm 0.08$ & $7.78 \pm 0.91$ & 1 \\
\hline NTTS $035120+3154$ NE & $\mathrm{x}$ & G5 & 5770 & 0.97 & $1.34_{-0.37}^{+0.64}$ & $I$ & $1.14 \pm 0.05$ & $7.43 \pm 0.08$ & 1 \\
\hline NTTS $040047+2603 E$ & $\mathrm{x}$ & M2 & 3580 & 0.49 & $0.25^{+0.26}$ & $I$ & $0.4 \pm 0.04$ & $6.4 \pm 0.19$ & 1 \\
\hline GSC 01262-00421 & $\mathrm{x}$ & F8 & 6200 & $0:$ & $4.82_{-1.15}^{+0.1}$ & $V$ & $1.44 \pm 0.05$ & $7.14 \pm 0.06$ & 2 \\
\hline RX J0405.7+2248 & $\mathrm{x}$ & G0 & 6030 & 0.02 & $5.47^{+1.15}$ & $V, I$ & $1.52 \pm 0.1$ & $7.07 \pm 0.08$ & 3 \\
\hline RX J0406.7+2018 & $\mathrm{x}$ & $\mathrm{F} 8$ & 6200 & 0.05 & $3.78^{+1.35}$ & $V, I$ & $1.39 \pm 0.06$ & $7.22 \pm 0.06$ & 3 \\
\hline V773 Tau A & o & $\mathrm{K} 2$ & 4900 & 1.32 & $2.34_{-0.16}^{+0.93}$ & $I$ & $1.66 \pm 0.05$ & $6.69 \pm 0.07$ & 4 \\
\hline V773 Tau B & o & K5 & 4350 & 1.32 & $0.85^{-0.16}+0.07$ & $I$ & $1.13 \pm 0.08$ & $6.71 \pm 0.1$ & 4 \\
\hline CW Tau & $\mathrm{c}$ & K3 & 4730 & 2.19 & $0.68^{-0.064}$ & $I$ & $1.11 \pm 0.15$ & $7.21 \pm 0.22$ & 1 \\
\hline FP Tau & c & M4 & 3370 & 0.24 & $0.51+0.27$ & $I$ & $0.31 \pm 0.03$ & $6.04 \pm 0.14$ & 1 \\
\hline CX Tau & $\mathrm{c}$ & $\mathrm{M} 2.5$ & 3580 & 0.83 & $0.56^{-0.15}+0.26$ & $I$ & $0.4 \pm 0.04$ & $6.12 \pm 0.09$ & 1 \\
\hline $\mathrm{LkCa} 4$ & o & K7 & 4060 & 0.69 & $0.73^{-0.15}+0.65$ & $I$ & $0.77 \pm 0.09$ & $6.43 \pm 0.25$ & 1 \\
\hline CY Tau & $\mathrm{c}$ & M1 & 3720 & 0.1 & $0.4_{-0.09}^{+0.09}$ & $I$ & $0.48 \pm 0.05$ & $6.37 \pm 0.11$ & 1 \\
\hline LkCa 5 & o & M2 & 3580 & 0.1 & $0.24_{-0.08}^{+0.07}$ & $I$ & $0.39 \pm 0.04$ & $6.5 \pm 0.17$ & 1 \\
\hline NTTS $041529+1652$ & $\mathrm{x}$ & K5 & 4350 & 0 & $0.15_{-0.07}^{+0.08}$ & $I$ & $0.85 \pm 0.19$ & $7.5 \pm 0.37$ & 1 \\
\hline V410 Tau ABC & o & K4 & 4730 & 0.03 & $1.41_{-0.29}^{+0.41}$ & $I$ & $1.45 \pm 0.09$ & $6.8 \pm 0.12$ & 1 \\
\hline DD Tau AB & $\mathrm{c}$ & M1 & 3720 & 1.61 & $\begin{array}{l}1.41-0.29 \\
0.61+0.33\end{array}$ & $I$ & $0.48 \pm 0.05$ & $6.16 \pm 0.13$ & 1 \\
\hline CZ Tau AB & $\mathrm{c}$ & M1.5 & 3650 & $\begin{array}{l}1.01 \\
1.32\end{array}$ & $0.6_{-0.017}^{+0.28}$ & $I$ & $0.43 \pm 0.04$ & $6.13 \pm 0.11$ & 1 \\
\hline Hubble 4 & o & K7 & 4060 & 0.76 & $0.48^{-0.17}+0.24$ & V & $0.79 \pm 0.08$ & $6.72 \pm 0.21$ & 1 \\
\hline NTTS $041559+1716$ & $\mathrm{x}$ & K7 & 4060 & 0 & $0.45^{-0.147}$ & $I$ & $0.79 \pm 0.08$ & $6.74 \pm 0.4$ & 1 \\
\hline BP Tau & c & K7 & 4060 & 0.49 & $0.65^{+0.13}$ & $I$ & $\begin{array}{l}0.78 \pm 0.00 \\
0.78 \pm 0.08\end{array}$ & $6.51 \pm 0.12$ & 1 \\
\hline V819 Tau AB & o & K7 & 4060 & 1.35 & $0.43^{-0.1}+0.14$ & $I$ & $0.8 \pm 0.08$ & $6.79 \pm 0.24$ & 1 \\
\hline $\mathrm{LkCa} 7 \mathrm{~A}$ & o & K7 & 4060 & 0.59 & $0.59_{-0.18}^{+0.32}$ & $\mathrm{~J}$ & $0.78 \pm 0.08$ & $6.57 \pm 0.21$ & 5 \\
\hline $\mathrm{LkCa} 7 \mathrm{~B}$ & o & M3.5 & 3420 & 0.59 & $0.18_{-0.05}^{+0.18}$ & $\mathrm{~J}$ & $0.31 \pm 0.03$ & $6.5 \pm 0.13$ & 5 \\
\hline RY Tau & c & $\mathrm{K} 1$ & 5080 & 1.84 & $6.59_{-1.04}^{+0.05}$ & $I$ & $2.24 \pm 0.07$ & $6.38 \pm 0.09$ & 1 \\
\hline HD 283572 & o & G5 & 5770 & 0.38 & $5.78_{-1.31}^{+1.94}$ & $I$ & $1.65 \pm 0.11$ & $6.94 \pm 0.08$ & 1 \\
\hline RX J0423.7+1537 & $\mathrm{x}$ & G5 & 5770 & 0.72 & $1_{-0.23}^{+0.36}$ & $V, I$ & 1.11: & 7.48: & 3 \\
\hline IP Tau & $\begin{array}{l}\hat{c} \\
\mathrm{c}\end{array}$ & M0 & 3850 & 0.24 & $0.34_{-0.12}^{+0.23}$ & $I$ & $0.59 \pm 0.07$ & $6.63 \pm 0.25$ & 1 \\
\hline DF Tau A & $\mathrm{c}$ & M1 & 3720 & 0.04 & $0.47_{-0.15}^{+0.30}$ & $I$ & $0.48 \pm 0.05$ & $6.28 \pm 0.17$ & 1,8 \\
\hline DF Tau B & c & M3.5 & 3420 & 0.04 & $0.53_{-0.18}^{+0.35}$ & $I$ & $0.32 \pm 0.03$ & $6.08 \pm 0.13$ & 1,8 \\
\hline NTTS $042417+1744$ & $\mathrm{x}$ & K1 & 5080 & $\begin{array}{c}0.4 \\
0.1\end{array}$ & $\begin{array}{l}1.99^{-0.18}+0.82 \\
1.99_{-01}\end{array}$ & $I$ & $1.51 \pm 0.14$ & $6.92 \pm 0.13$ & $\begin{array}{l}1,0 \\
1\end{array}$ \\
\hline DI Tau AB & $\begin{array}{l}\hat{\alpha} \\
\mathrm{o}\end{array}$ & M0 & 3850 & 0.76 & $0.85_{-0.035}^{+0.91}$ & $I$ & $0.56 \pm 0.06$ & $6.11 \pm 0.21$ & 1 \\
\hline IQ Tau & $\mathrm{c}$ & M0.5 & 3785 & 1.25 & $0.53+0.35$ & $I$ & $0.52 \pm 0.05$ & $6.28 \pm 0.21$ & 1 \\
\hline UX Tau A & c & K5 & 4350 & 0 & $1.29^{-0.2}+0.5$ & $I$ & $1.12 \pm 0.13$ & $6.43 \pm 0.17$ & 1 \\
\hline UX Tau B & o & M2 & 3580 & 0 & $0.15^{-0.032}$ & $\mathrm{~J}$ & $0.38 \pm 0.04$ & $6.75 \pm 0.17$ & $\begin{array}{l}1 \\
5\end{array}$ \\
\hline FX Tau AB & $\mathrm{c}$ & M1 & 3720 & 2.24 & $0.89^{-0.04}+0.7$ & $I$ & $0.47 \pm 0.05$ & $6.05 \pm 0.1$ & 1 \\
\hline DK Tau AB & c & K7 & 4060 & 0.35 & $0.9+0.32$ & $\begin{array}{l}I \\
I\end{array}$ & $0.76 \pm 0.08$ & $6.31 \pm 0.13$ & 1 \\
\hline V927 Tau AB & c & M3 & 3470 & 1.4 & $0.6_{-0.22}^{-0.21}$ & $I$ & $0.35 \pm 0.03$ & $6.04 \pm 0.18$ & 1 \\
\hline NTTS $042835+1700$ & $\mathrm{x}$ & K5 & 4350 & 0.21 & $0.19_{-0.08}^{+0.229}$ & $I$ & $0.82 \pm 0.09$ & $7.57 \pm 0.24$ & 1 \\
\hline HK Tau AB & $\mathrm{c}$ & M0.5 & 3785 & 3.41 & $1_{-0.49}^{+1.9808}$ & $I$ & $0.52 \pm 0.05$ & $5.97 \pm 0.27$ & 1 \\
\hline $\mathrm{L} 1551-51 \mathrm{AB}$ & o & $\mathrm{K} 7$ & 4060 & 0 & $0.49^{-0.49}+0.14$ & $I$ & $0.79 \pm 0.08$ & $6.71 \pm 0.16$ & 1 \\
\hline V827 Tau & o & K7 & 4060 & 0.28 & $0.87^{+0.45}$ & $I$ & $0.76 \pm 0.08$ & $6.33 \pm 0.17$ & 1 \\
\hline V826 Tau AB & o & K7 & 4060 & 0.08 & $0.5_{-0.09}^{+0.22^{6}}$ & $I$ & $0.79 \pm 0.08$ & $6.69 \pm 0.14$ & 1 \\
\hline V928 Tau AB & o & M0.5 & 3785 & 1.87 & $0.82^{-0.09}+0.64$ & $I$ & $0.52 \pm 0.05$ & $6.07 \pm 0.16$ & 1 \\
\hline RX J0432.8+1735 & $\mathrm{x}$ & M2 & 3580 & 0.65 & $0.52_{-0.74}^{+0.29}$ & $V, J$ & $0.4 \pm 0.04$ & $6.14 \pm 0.2$ & 3 \\
\hline GG Tau Aa & $\mathrm{c}$ & K7 & 4060 & 0.7 & $0.64^{+0.24}$ & $I$ & $0.78 \pm 0.08$ & $6.52 \pm 0.13$ & 6 \\
\hline GG Tau Ab & $\mathrm{c}$ & M0.5 & 3720 & 3.2 & $0.57^{+0.112}$ & $I$ & $0.48 \pm 0.05$ & $6.19 \pm 0.09$ & 6 \\
\hline GH Tau AB & $\mathrm{c}$ & M2 & 3580 & 1.04 & $0.98_{-0.34}^{+0.11}$ & $I$ & $0.4 \pm 0.03$ & $5.82 \pm 0.32$ & 1 \\
\hline DM Tau & $\mathrm{c}$ & M1 & 3720 & 0 & $0.16^{+0.34}$ & $I$ & $0.47 \pm 0.06$ & $6.87 \pm 0.34$ & 1 \\
\hline CI Tau & c & K7 & 4060 & 1.77 & $0.96_{-0.44}^{+0.36}$ & $I$ & $0.76 \pm 0.08$ & $6.27 \pm 0.28$ & 1 \\
\hline NTTS $043124+1824$ & $\mathrm{x}$ & G8 & 5520 & 1.08 & $2.93+33_{-122}^{+3.24}$ & $I$ & $1.55 \pm 0.22$ & $7 \pm 0.16$ & 1 \\
\hline V1078 Tau & $\mathrm{x}$ & $\mathrm{F} 8$ & 6200 & 0.94 & $5.82+2.91$ & $V, J$ & $1.53 \pm 0.11$ & $7.09 \pm 0.09$ & 2 \\
\hline DN Tau & $\mathrm{c}$ & M0 & 3850 & 0.49 & $0.79^{-1.622}$ & $I$ & $0.57 \pm 0.06$ & $6.15 \pm 0.11$ & 1 \\
\hline $\mathrm{LkCa} 14$ & o & M0 & 3850 & 0 & $0.76^{-0.16}$ & $I$ & $0.57 \pm 0.06$ & $6.16 \pm 0.11$ & 1 \\
\hline DO Tau & $\mathrm{c}$ & M0 & 3850 & 2.64 & $1.29^{-0.15}$ & $I$ & $0.56 \pm 0.05$ & $5.93 \pm 0.15$ & 1 \\
\hline HV Tau AB & c & M1 & 3720 & 2.42 & $0.6_{-0.14}^{+0.42}$ & $I$ & $0.48 \pm 0.05$ & $6.17 \pm 0.11$ & 1 \\
\hline VY Tau AB & $\mathrm{c}$ & M0 & 3850 & 0.38 & $0.3^{-0.14}$ & $I$ & $0.59 \pm 0.07$ & $6.7 \pm 0.3$ & 1 \\
\hline $\mathrm{LkCa} 15$ & $\mathrm{c}$ & K5 & 4350 & 0.62 & $0.85^{-0.12}+0.3$ & $I$ & $1.12 \pm 0.08$ & $6.7 \pm 0.16$ & 1 \\
\hline IW Tau AB & o & K7 & 4060 & 0.83 & $0.99^{+0.7^{+0.2}}$ & $I$ & $0.75 \pm 0.08$ & $6.26 \pm 0.19$ & 1 \\
\hline $\mathrm{LkH} \alpha 332 \mathrm{G} 2 \mathrm{AB}$ & o & K7 & 4060 & 3.16 & $0.49^{-0.34}$ & $\mathrm{~V}$ & $0.79 \pm 0.08$ & $6.7 \pm 0.24$ & 1 \\
\hline GO Tau & c & M0 & 3850 & 1.18 & $0.22_{-0.08}^{+0.16}$ & $I$ & $0.59 \pm 0.07$ & $6.91 \pm 0.28$ & 1 \\
\hline DR Tau & $\mathrm{c}$ & K7 & 4060 & 2: & $1.97_{-0.92}^{+3.08}$ & $I$ & $0.74 \pm 0.08$ & $5.92 \pm 0.23$ & 1 \\
\hline
\end{tabular}


C. Bertout et al.: The evolution of stars in the Taurus-Auriga T association, Online Material p 3

Table 4. continued.

\begin{tabular}{|c|c|c|c|c|c|c|c|c|c|}
\hline Star & Type $^{1}$ & Sp. type & $\bar{T} T_{\text {eff }}$ & $A_{\mathrm{V}}$ & $L_{\text {star }} / L_{\odot}$ & Filter $^{2}$ & $M_{\text {star }} / M_{\odot}$ & $\log t \pm \sigma_{\log t}(t$ in $\mathrm{yr})$ & Ref. $^{3}$ \\
\hline UY Aur AB & $\mathrm{c}$ & K7 & 4060 & 2.05 & $1.41_{-0.35}^{+0.57}$ & $I$ & $0.74 \pm 0.07$ & $6.07 \pm 0.11$ & 1 \\
\hline RX J0452.5+1730 & $\mathrm{x}$ & K4 & 4590 & $0:$ & $0.39_{-0.18}^{+0.59}$ & $V$ & $0.99 \pm 0.17$ & $7.3 \pm 0.27$ & 3 \\
\hline GM Aur & $\mathrm{c}$ & $\mathrm{K} 3$ & 4730 & 0.14 & $1.23_{-0.47}^{+1.07}$ & $I$ & $1.37 \pm 0.17$ & $6.87 \pm 0.23$ & 1 \\
\hline LkCa 19 & o & K0 & 5250 & 0 & $1.56_{-0.34}^{+0.5^{\prime}}$ & $I$ & $1.28 \pm 0.1$ & $7.17 \pm 0.1$ & 1 \\
\hline AB Aur & $\mathrm{a}$ & B9 & 10500 & 0.62 & $44.86_{-12}^{+20}$ & $I$ & $2.58 \pm 0.14$ & $6.68 \pm 0.08$ & 1 \\
\hline SU Aur & $\mathrm{c}$ & $\mathrm{G} 2$ & 5860 & 0.9 & $9.29_{-1.65}^{+2.26}$ & $I$ & $1.88 \pm 0.1$ & $6.8 \pm 0.08$ & 1 \\
\hline NTTS $045251+3016$ AB & $\mathrm{x}$ & K7 & 4060 & 0 & $0.54_{-0.1}^{+0.14}$ & $I$ & $0.79 \pm 0.08$ & $6.64 \pm 0.14$ & 7 \\
\hline RX J0457.0+1517 & $\mathrm{x}$ & G0 & 6030 & 0.75 & $1.75_{-0.0}^{+0.6}$ & $V, J$ & $1.21 \pm 0$ & $7.55 \pm 0.31$ & 3 \\
\hline RX J0457.5+2014 AB & $\mathrm{x}$ & K3 & 4730 & 0.05 & $0.86_{-0.2}^{+0.31}$ & $V, J$ & $1.22 \pm 0.11$ & $7.09 \pm 0.13$ & 3 \\
\hline RW Aur A & $\mathrm{c}$ & K4 & 4590 & 0.32 & $1.72_{-0.33}^{+0.47}$ & $I$ & $1.07 \pm 0.06$ & $7.20 \pm 0.11$ & 1,8 \\
\hline
\end{tabular}

${ }^{1}$ Meaning of symbols: $\mathrm{c}=$ CTTS, $\mathrm{o}=$ optically selected WTTS, $\mathrm{x}=\mathrm{X}$-ray selected WTTS.

${ }^{2}$ Photometric filter used for determining the photospheric luminosity. $I$ stands for the Cousins $I$ filter, $J$ for the 2 MASS $J$ filter. When 2 filters are given, $A_{\mathrm{V}}$ is computed by assuming that the photospheric luminosities using both filters are equal.

${ }^{3}$ References for observational properties: (1) Kenyon \& Hartmann (1995); (2) Walter et al. (1988); (3) Wichmann et al. (2000); (4) Welty (1995); (5) Woitas et al. (2001); (6) White et al. (1999); (7) Leinert et al. (1993); (8) Ghez et al. (1997). 